\title{
Cattle Hypomagnesemia, Puerperal Tetany and Mg/Ca Ratio of Cropland and Groundwater
}

\author{
Töysä T* \\ Specialty General Practice, Student of University of Eastern Finland, Finland \\ *Corresponding author: Töysä T, Specialty General Practice, Student of University of Eastern Finland, Finland
}

\begin{tabular}{|c|c|}
\hline ARTICLE INFO & ABSTRACT \\
\hline Received: 蔧 July 02, 2020 & Citation: Töysä T. Cattle Hypomagnesemia, Puerperal Tetany and $\mathrm{Mg} / \mathrm{Ca}$ Ratio of \\
\hline Published: 慧 July 16, 2020 & pland and Groundwater. Blomed J Scl \& Iech Res $28(5)-2020$. BJSTR. MS.ID.004 \\
\hline
\end{tabular}

\section{Mini Review}

Cattle hypomagnesemia has been typically a pasture disease and labeled "grass tetany", but after its increase during indoor periods only single label "hypomagnesemia" has been used after 1983. Cropland and groundwater (gw) Mg/Ca ratio in Finnish Rural Centers (RC) [1]. Northern RC's are MK11, MK16, 17, 18 and 19 are labeled with gray color. Agricultural soil $\mathrm{Mg} / \mathrm{Ca}$ is higher in North than in In Other parts of Finland (with and without exclusion of Åland), but not in groundwater. Numbers of Finnish Provinces are from [2]. Data on veterinary monthly reports by provinces are only partially available [3], concerning December 1979, December 1981,
December 1985, December 1989, December 1991, December 1992, December 1993, Year 1994, Year 1995 and Year 1996. Provinces are in order of the Rural Centers labeled by the numbers of [2]: given by abbreviations since 1991: 01 Uudenmaan - UL, 02 Turun ja Porin - T-PL, 03 Ahvenanmaa - ÅL (Åland), 04 Hämeen - HL, 05 Kymen - KYL, 06 Mikkelin - ML, 08 Kuopion - KUL, 07 Pohjois-Karjalan P-KL, 09 Keski-Suomen - K-SL, 10 Vaasan - VL, 11 Oulun - OL, 12 Lapin - LL. About 1/3 of MK16 Central Ostrobothnia in belonging to "Vaasan" [4], but here "Vaasan" has been treated as a pure part of South Finland (Figures 1 \& 2) (Tables $1 \& 2$ ).

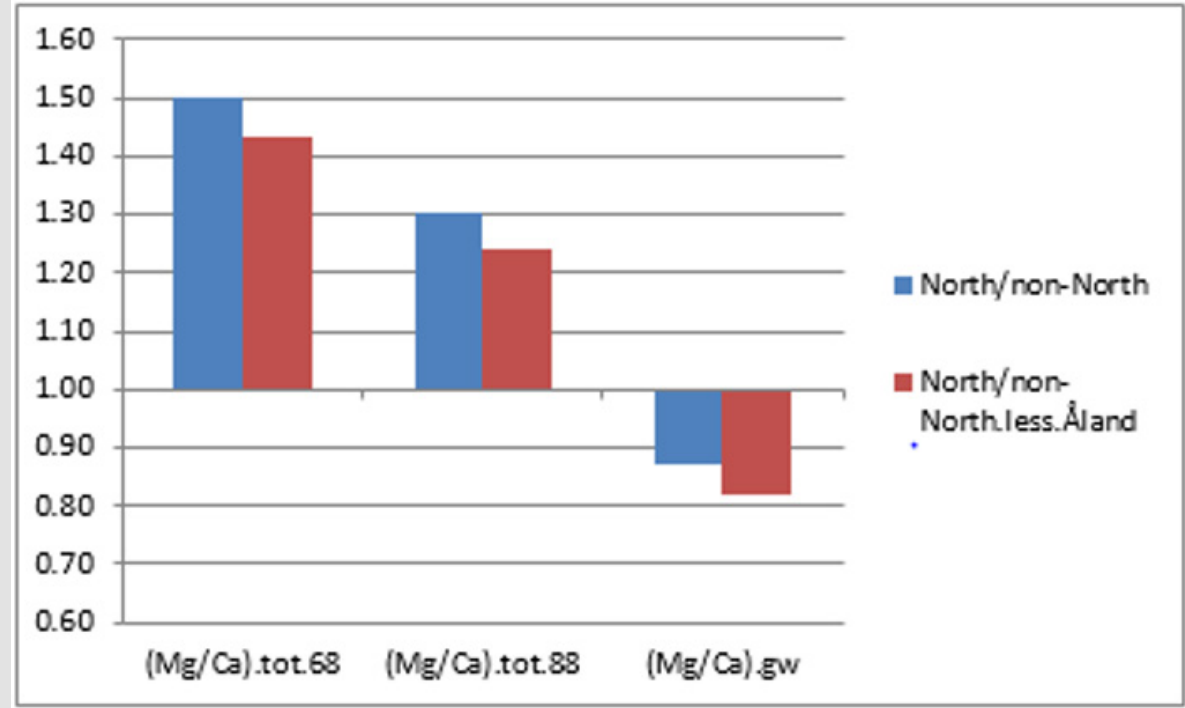

Figure 1. 


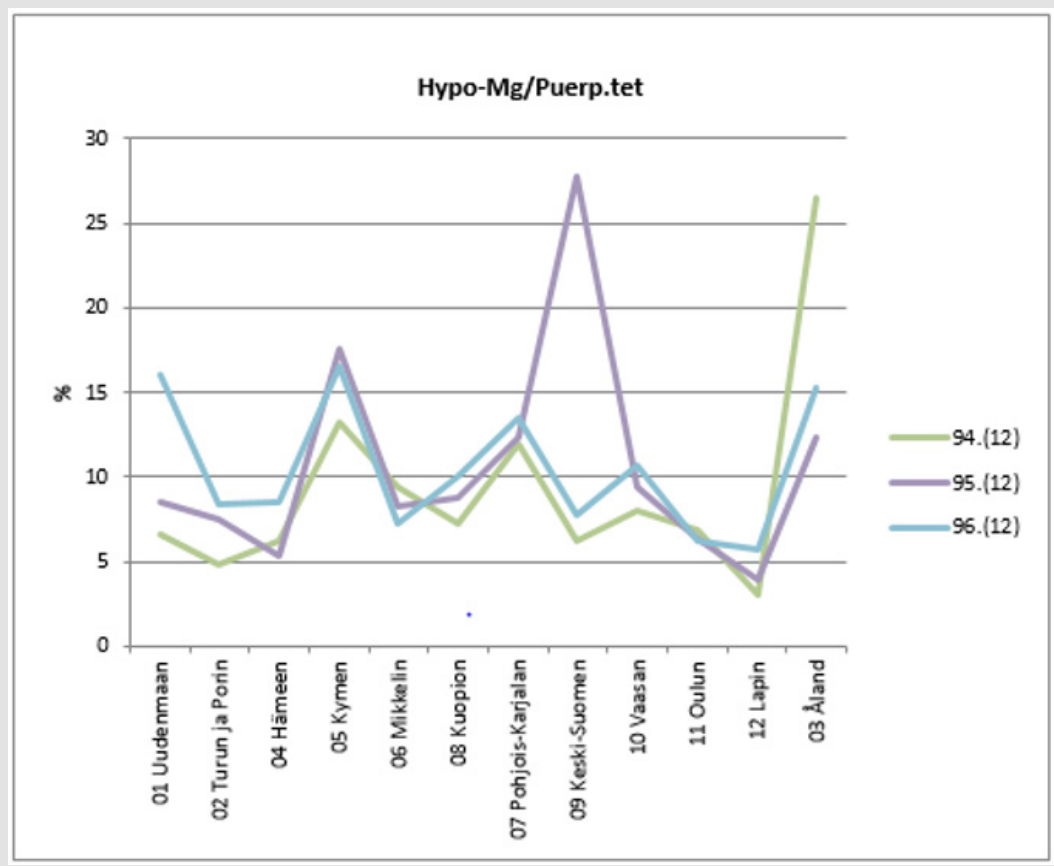

Figure 2.

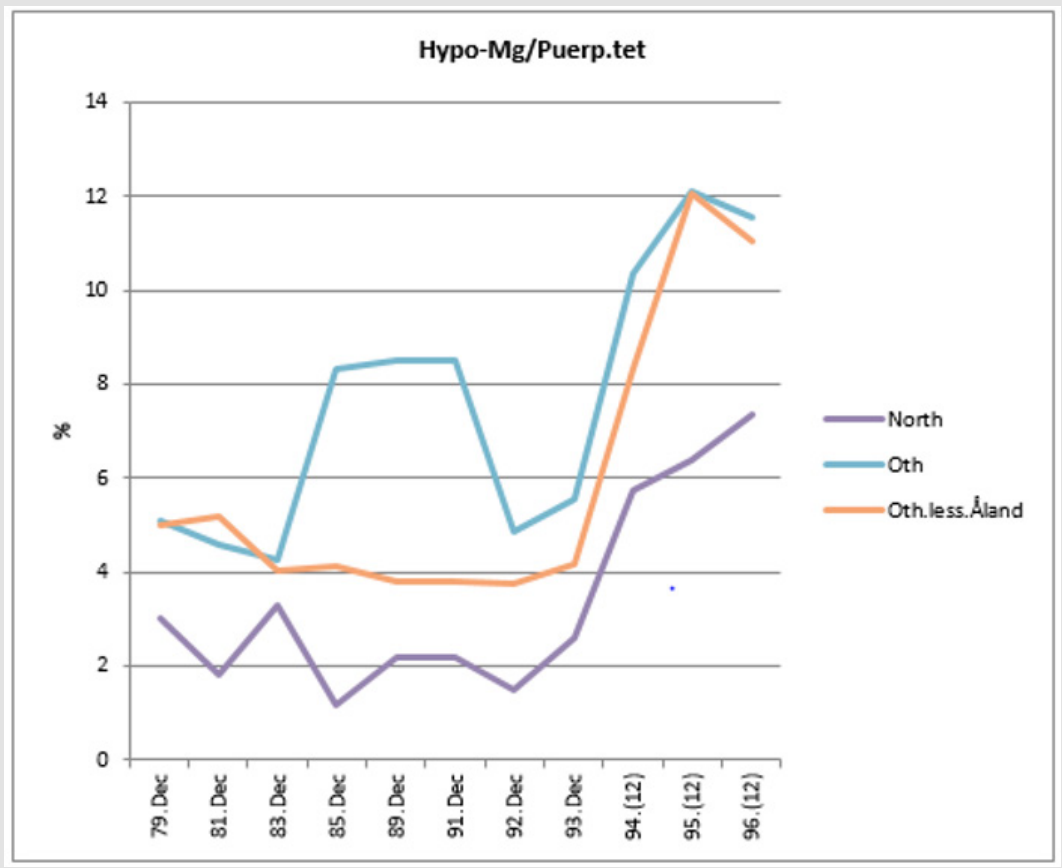

Figure 3.

Table 1.

\begin{tabular}{|c|c|c|c|}
\hline & $\mathbf{( M g} /$ Ca).tot.68 & $\mathbf{( M g} / \mathbf{C a})$. tot.88 & (Mg/Ca).gw \\
\hline MK01 Uusimaa & 0.27 & 0.312 & 0.53 \\
\hline MK02 Varsinais-Suomi & 0.282 & 0.26 & 0.58 \\
\hline MK04 Satakunta & 0.181 & 0.222 & 0.35 \\
\hline MK05 Kanta-Häme & 0.286 & 0.296 & 0.61 \\
\hline MK06 Pirkanmaa & 0.209 & 0.227 & 0.45 \\
\hline MK07 Päijät-Häme & 0.176 & 0.203 & 0.41 \\
\hline MK08 Kymenlaakso & 0.232 & 0.288 & 0.41 \\
\hline
\end{tabular}




\begin{tabular}{|c|c|c|c|}
\hline MK09 South Karelia & 0.157 & 0.191 & 0.39 \\
\hline MK10 Etelä-Savo & 0.152 & 0.167 & 0.29 \\
\hline MK11 Pohjois-Savo & 0.231 & 0.234 & 0.32 \\
\hline MK12 North Karelia & 0.165 & 0.214 & 0.33 \\
\hline MK13 Central Finland & 0.202 & 0.221 & 0.32 \\
\hline MK14 South Ostrobothnia & 0.21 & 0.258 & 0.36 \\
\hline MK15 Ostrobothnia & 0.234 & 0.267 & 0.46 \\
\hline MK16 Central Ostrobothnia & 0.272 & 0.296 & 0.34 \\
\hline MK17 North Ostrobothnia & 0.349 & 0.333 & 0.35 \\
\hline MK18 Kainuu & 0.324 & 0.292 & 0.32 \\
\hline MK19 Lapland & 0.341 & 0.339 & 0.41 \\
\hline MK21 Åland & 0.073 & 0.081 & 0.08 \\
\hline North & 0.303 & 0.299 & 0.347 \\
\hline SW.less.Åland & 0.216 & 0.241 & 0.447 \\
\hline SE & 0.203 & 0.24 & 0.369 \\
\hline (SW+SE).less.Åland & 0.212 & 0.241 & 0.423 \\
\hline Åland & 0.073 & 0.081 & 0.081 \\
\hline non-North & 0.202 & 0.229 & 0.398 \\
\hline
\end{tabular}

Table 2.

\begin{tabular}{|c|c|c|c|c|c|c|c|c|c|c|c|c|}
\hline \multicolumn{13}{|c|}{ Hypomagnesemia } \\
\hline & $\begin{array}{c}01 \\
\text { Uudenmaan }\end{array}$ & $\begin{array}{c}02 \\
\text { Turun } \\
\text { ja Porin }\end{array}$ & $\begin{array}{c}04 \\
\text { Hämeen }\end{array}$ & $\begin{array}{c}05 \\
\text { Kymen }\end{array}$ & $\begin{array}{c}06 \\
\text { Mikkelin }\end{array}$ & $\begin{array}{c}08 \\
\text { Kuopion }\end{array}$ & $\begin{array}{c}07 \\
\text { Pohjois- } \\
\text { Karjalan }\end{array}$ & $\begin{array}{c}09 \\
\text { Keski- } \\
\text { Suomen }\end{array}$ & $\begin{array}{c}10 \\
\text { Vaasan }\end{array}$ & $\begin{array}{c}11 \\
\text { Oulun }\end{array}$ & $\begin{array}{c}12 \\
\text { Lapin }\end{array}$ & $\begin{array}{c}03 \\
\text { Åland }\end{array}$ \\
\hline 79.Dec & 38 & 25 & 21 & 10 & 8 & 6 & 6 & 12 & 19 & 25 & 4 & 1 \\
\hline 81.Dec & 25 & 22 & 10 & 23 & 4 & 2 & 21 & 3 & 16 & 14 & 1 & 0 \\
\hline 83.Dec & 33 & 19 & 16 & 12 & 7 & 15 & 1 & 5 & 10 & 13 & 4 & 1 \\
\hline 85.Dec & 29 & 14 & 8 & 12 & 4 & 5 & 15 & 0 & 24 & 8 & 0 & 5 \\
\hline 89.Dec & 6 & 7 & 16 & 18 & 6 & 8 & 6 & 0 & 23 & 7 & 1 & 6 \\
\hline 91.Dec & 6 & 7 & 16 & 18 & 6 & 8 & 6 & 0 & 23 & 7 & 1 & 6 \\
\hline 92.Dec & 2 & 8 & 10 & 16 & 5 & 1 & 8 & 1 & 7 & 11 & 0 & 2 \\
\hline 93.Dec & 8 & 8 & 6 & 27 & 4 & 5 & 10 & 0 & 15 & 16 & 2 & 2 \\
\hline 94.(12) & 117 & 131 & 222 & 317 & 189 & 298 & 252 & 98 & 436 & 336 & 28 & 27 \\
\hline 95.(12) & 117 & 164 & 149 & 314 & 155 & 325 & 198 & 365 & 432 & 281 & 31 & 18 \\
\hline 96.(12) & 201 & 154 & 226 & 243 & 132 & 357 & 206 & 105 & 482 & 258 & 40 & 17 \\
\hline
\end{tabular}

Table 3.

\begin{tabular}{|c|c|c|c|c|c|c|c|c|c|c|c|c|}
\hline \multicolumn{13}{|c|}{ Puerperal tet } \\
\hline & $\begin{array}{c}01 \\
\text { Uudenmaan }\end{array}$ & $\begin{array}{c}02 \\
\text { Turun } \\
\text { ja Porin }\end{array}$ & $\begin{array}{c}04 \\
\text { Hämeen }\end{array}$ & $\begin{array}{c}05 \\
\text { Kymen }\end{array}$ & $\begin{array}{c}06 \\
\text { Mikkelin }\end{array}$ & $\begin{array}{c}08 \\
\text { Kuopion }\end{array}$ & $\begin{array}{c}07 \\
\text { Pohjois- } \\
\text { Karjalan } \\
\end{array}$ & $\begin{array}{c}09 \\
\text { Keski- } \\
\text { Suomen }\end{array}$ & $\begin{array}{c}10 \\
\text { Vaasan }\end{array}$ & $\begin{array}{c}11 \\
\text { Oulun }\end{array}$ & $\begin{array}{c}12 \\
\text { Lapin }\end{array}$ & $\begin{array}{c}03 \\
\text { Åland }\end{array}$ \\
\hline 79.Dec & 351 & 444 & 456 & 303 & 253 & 448 & 295 & 161 & 608 & 520 & 136 & 18 \\
\hline 81.Dec & 253 & 400 & 324 & 337 & 236 & 305 & 219 & 173 & 529 & 397 & 81 & 23 \\
\hline 83.Dec & 348 & 413 & 421 & 278 & 170 & 446 & 200 & 142 & 533 & 446 & 112 & 16 \\
\hline 85.Dec & 291 & 367 & 335 & 290 & 204 & 373 & 213 & 203 & 654 & 367 & 47 & 12 \\
\hline 89.Dec & 219 & 284 & 337 & 177 & 222 & 306 & 223 & 115 & 466 & 311 & 59 & 13 \\
\hline 91.Dec & 219 & 284 & 337 & 177 & 222 & 306 & 223 & 115 & 466 & 311 & 59 & 13 \\
\hline 92.Dec & 173 & 224 & 213 & 173 & 156 & 255 & 144 & 120 & 367 & 267 & 63 & 15 \\
\hline 93.Dec & 184 & 214 & 339 & 230 & 219 & 411 & 159 & 167 & 415 & 363 & 90 & 12 \\
\hline 94.(12) & 1755 & 2687 & 3549 & 2393 & 2008 & 4088 & 2112 & 1586 & 5445 & 4861 & 929 & 102 \\
\hline 95.(12) & 1384 & 2206 & 2826 & 1791 & 1883 & 3711 & 1604 & 1317 & 4569 & 4434 & 778 & 146 \\
\hline 96.(12) & 1256 & 1849 & 2668 & 1465 & 1836 & 3559 & 1529 & 1365 & 4498 & 4111 & 697 & 111 \\
\hline
\end{tabular}


Table 4.

\begin{tabular}{|c|c|c|c|c|c|c|c|c|c|c|c|c|}
\hline \multicolumn{13}{|c|}{ (Hypo-Mg/Puerp.tet) \% } \\
\hline & $\begin{array}{c}01 \\
\text { Uudenmaan }\end{array}$ & $\begin{array}{c}02 \\
\text { Turun } \\
\text { ja Porin }\end{array}$ & $\begin{array}{c}04 \\
\text { Hämeen }\end{array}$ & $\begin{array}{c}05 \\
\text { Kymen }\end{array}$ & $\begin{array}{c}06 \\
\text { Mikkelin }\end{array}$ & $\begin{array}{c}08 \\
\text { Kuopion }\end{array}$ & $\begin{array}{c}07 \\
\text { Pohjois- } \\
\text { Karjalan }\end{array}$ & $\begin{array}{c}09 \\
\text { Keski- } \\
\text { Suomen }\end{array}$ & $\begin{array}{c}10 \\
\text { Vaasan }\end{array}$ & $\begin{array}{c}11 \\
\text { Oulun }\end{array}$ & $\begin{array}{c}12 \\
\text { Lapin }\end{array}$ & $\begin{array}{c}03 \\
\text { Åland }\end{array}$ \\
\hline 79.Dec & 10.8 & 5.6 & 4.6 & 3.3 & 3.2 & 1.3 & 2 & 7.5 & 3.1 & 4.8 & 2.9 & 5.6 \\
\hline 81.Dec & 9.9 & 5.5 & 3.1 & 6.8 & 1.7 & 0.7 & 9.6 & 1.7 & 3 & 3.5 & 1.2 & 0 \\
\hline 83.Dec & 9.5 & 4.6 & 3.8 & 4.3 & 4.1 & 3.4 & 0.5 & 3.5 & 1.9 & 2.9 & 3.6 & 6.3 \\
\hline 85.Dec & 10 & 3.8 & 2.4 & 4.1 & 2 & 1.3 & 7 & 0 & 3.7 & 2.2 & 0 & 41.7 \\
\hline 89.Dec & 2.7 & 2.5 & 4.7 & 10.2 & 2.7 & 2.6 & 2.7 & 0 & 4.9 & 2.3 & 1.7 & 46.2 \\
\hline 91.Dec & 2.7 & 2.5 & 4.7 & 10.2 & 2.7 & 2.6 & 2.7 & 0 & 4.9 & 2.3 & 1.7 & 46.2 \\
\hline 92.Dec & 1.2 & 3.6 & 4.7 & 9.2 & 3.2 & 0.4 & 5.6 & 0.8 & 1.9 & 4.1 & 0 & 13.3 \\
\hline 93.Dec & 4.3 & 3.7 & 1.8 & 11.7 & 1.8 & 1.2 & 6.3 & 0 & 3.6 & 4.4 & 2.2 & 16.7 \\
\hline 94.(12) & 6.7 & 4.9 & 6.3 & 13.2 & 9.4 & 7.3 & 11.9 & 6.2 & 8 & 6.9 & 3 & 26.5 \\
\hline 95.(12) & 8.5 & 7.4 & 5.3 & 17.5 & 8.2 & 8.8 & 12.3 & 27.7 & 9.5 & 6.3 & 4 & 12.3 \\
\hline 96.(12) & 16 & 8.3 & 8.5 & 16.6 & 7.2 & 10 & 13.5 & 7.7 & 10.7 & 6.3 & 5.7 & 15.3 \\
\hline
\end{tabular}

\section{Discussion}

Increase of Hypo-Mg/Puerperal tetany ratio after 1993 shows that hypomagnesemia is still more common during pasture period. It can even depend on several other factors: e.g. variation of weather, temperature and grass digestibility (incl sugars) (Tables $3 \& 4$ ).

In closer analysis is seen one discrepancy: HMg associates in 1979, 1981 \& 1983 (slightly) positively with (Mg/Ca).soil, even by using linearly interpolated $(\mathrm{Mg} / \mathrm{Ca}$ ) ratios (calculated by values from 1976-80 (.78) and 1986- 80 (.88). This needs a new assessment. Data on period 1979- 83 should be opened.

Phosphor and obvious association of hyperirritative syndromon with HMg seems to need a closer assessment, too.

\section{Conclusion}

Ratio of cattle hypomagnesemia cases per cases of puerperal paresis in monthly reports of veterinary surgeons were constantly lower in North Finland than in other parts of Finland, where $\mathrm{Mg} / \mathrm{Ca}$ ratio of soil was lower (1965-85), but groundwater $\mathrm{Mg} / \mathrm{Ca}$ higher. This suggests on higher importance of cropland than gw $\mathrm{Mg} / \mathrm{Ca}$ ratio in livestock and human nutrition.

\section{References}

1. Regional Groundwater $\mathrm{Si}$ and $\mathrm{Mg} / \mathrm{Ca}$, Soil $\mathrm{Mg} / \mathrm{Ca}$, Soil-Types, Cardiac Diseases and Local Long-Term Dolomite Application - Soil Values from Two Periods Suggest on Different Roles of Mg and Si in Cardiac Epidemiology. Biomed J Sci \& Tech Res 26(5): 20363.

2. Statistical Yearbook of Finland 1980. Tilastokeskus (1981) Helsinki (map of Finnish provinces down left on the page XXX).

3. I-XI. Incidence of some animal diseases according monthly veterinary reports. ("Eräiden eläintautien esiintyminen Suomessa eläinlääkäreiden kuukausi-ilmoitusten mukaan") [In Finnish].

4. Toysa T, Osmo Hanninen (2016) Soil Weathering, Silicon and CHD in Finland. J J Agriculture 2(1): 008.
ISSN: 2574-1241

DOI: $10.26717 /$ BJSTR.2020.28.004725

Töysä T. Biomed J Sci \& Tech Res

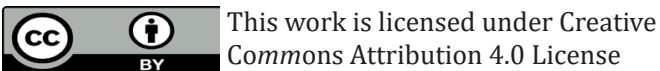

Submission Link: https://biomedres.us/submit-manuscript.php

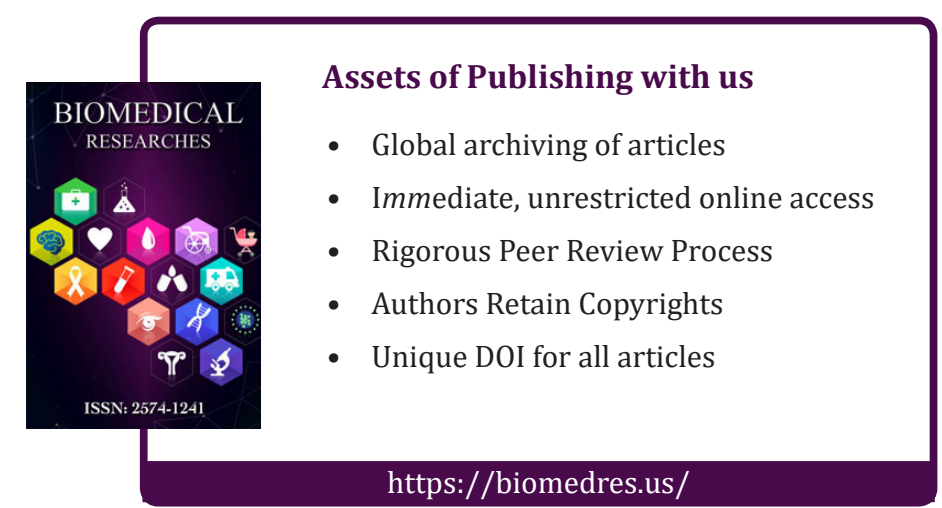

